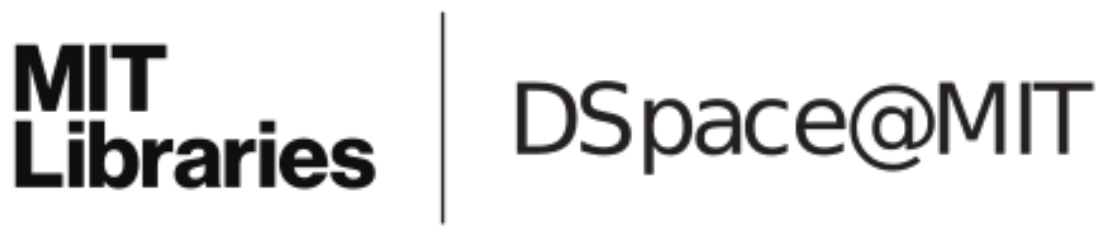

\author{
MIT Open Access Articles
}

Non-reciprocity in nonlinear elastodynamics

The MIT Faculty has made this article openly available. Please share how this access benefits you. Your story matters.

Citation: Blanchard, Antoine, Themistoklis P. Sapsis and Alexander F. Vakakis. "Non-Reciprocity in Nonlinear Elastodynamics." Journal of Sound and Vibration 412 (January 2018): pages 326-335.

As Published: 10.1016/j.jsv.2017.09.039

Publisher: Elsevier BV

Persistent URL: https://hdl.handle.net/1721.1/121935

Version: Original manuscript: author's manuscript prior to formal peer review

Terms of use: Creative Commons Attribution-NonCommercial-NoDerivs License 


\title{
Non-Reciprocity in Nonlinear Elastodynamics
}

\author{
Antoine Blanchard ${ }^{\mathrm{a}, *}$, Themistoklis P. Sapsis ${ }^{\mathrm{b}}$, Alexander F. Vakakis ${ }^{\mathrm{c}}$ \\ ${ }^{a}$ Department of Aerospace Engineering, University of Illinois, \\ Urbana, IL 61801, USA \\ ${ }^{b}$ Department of Mechanical Engineering, Massachussetts Institute of Technology, \\ Cambridge, MA 02139, USA \\ ${ }^{c}$ Department of Mechanical Science and Engineering, University of Illinois, \\ Urbana, IL 61801, USA
}

\begin{abstract}
Reciprocity is a fundamental property of linear time-invariant (LTI) acoustic waveguides governed by self-adjoint operators with symmetric Green's functions. The break of reciprocity in LTI elastodynamics is only possible through the break of time reversal symmetry on the micro-level, and this can be achieved by imposing external biases, adding nonlinearities or allowing for time-varying system properties. We present a Volterra-series based asymptotic analysis for studying spatial non-reciprocity in a class of one-dimensional (1D), time-invariant elastic systems with weak stiffness nonlinearities. We show that nonlinearity is neither necessary nor sufficient for breaking reciprocity in this class of systems; rather, it depends on the boundary conditions, the symmetries of the governing linear and nonlinear operators, and the choice of the spatial points where the non-reciprocity criterion is tested. Extension of the analysis to higher dimensions and time-varying systems is straightforward from a mathematical point of view (but not in terms of new non-reciprocal physical phenomena), whereas the connection of non-reciprocity and time irreversibility can be studied as well. Finally, we show that suitably defined non-reciprocity measures enable optimization, and can provide physical understanding of the nonlinear effects in the dynamics, enabling one to establish regimes of "maximum nonlinearity". We
\end{abstract}

\footnotetext{
* Corresponding author

Email addresses: ablancha@illinois.edu (Antoine Blanchard), sapsis@mit.edu (Themistoklis P. Sapsis), avakakis@illinois.edu (Alexander F. Vakakis)
} 
highlight the theoretical developments by means of a numerical example.

Keywords: nonlinear, reciprocity, elastodynamics

\section{Introduction}

Reciprocity is a well-known and fundamental property of linear time-invariant (LTI) acoustic waveguides 1, 2, going back to the work of Lord Rayleigh on the theory of sound. In structural acoustics, reciprocity is a fundamental property 5 of LTI elastic systems governed by self-adjoint operators, leading to symmetric Green's functions [3. For these media (but also, more generally, in linear or linearized mechanics) the Betti-Maxwell reciprocity theorem applies. In a broader context, in LTI (possibly inhomogeneous) waveguides, reciprocity is directly related to time-reversal symmetry through the Onsager-Casimir principle of microscopic reversibility 4 . 6 . Accordingly, the break of reciprocity in LTI elastodynamics is only possible through the break of time reversal symmetry on the micro-level [7, but not necessarily on the macro-level. For example, in linear absorbing media with linear viscous dissipation, although time reversal symmetry is broken on the macro-level, reciprocity is still preserved since time 15 reversibility holds on the micro-level.

One way to break reciprocity in LTI elastic systems is by introducing an oddsymmetric external bias, for example, a unidirectional static magnetic field, or a unidirectional fluid circulation. An example was given by Fleury et al. [8], where an acoustic analog of the Zeeman electromagnetic non-reciprocal phenomenon was applied to a resonant ring acoustic cavity biased by a circulating fluid with a constant direction of rotation. This led to giant non-reciprocity (that is, to complete elimination of reciprocity), and resulted in preferential sound transmission [9]. Tsakmakidis et al. [10, proposed a broadband highQ optical cavity connected to a semiconductor-dielectric-metal waveguide by breaking reciprocity of light propagation by means of an external unidirectional magnetic field. This changes the widely-held view that any type of system having a given bandwidth can interact with an incident wave only over a restricted 
time period which is inversely proportional to the bandwidth.

Alternative ways to break the conditions of the Onsager-Casimir principle and induce non-reciprocity is by introducing nonlinearities or time-variant properties. An example of the latter approach was given by Fleury et al. 11] with coupled acoustic cavities whose volumes were harmonically modulated, imparting an effective angular momentum bias. Another approach employed nonlinear active metamaterials [12. Regarding nonlinear acoustic non-reciprocity [13,

35 Liang et al. 14, 15, proposed an acoustic diode by coupling a superlattice with a nonlinear medium, whereas Boechler et al. [16] and Li et al. [17] used granular media for nonlinear acoustic switching, rectification and logic. Finally, Zhang et al. [18 studied nonlinear non-reciprocity phenomena in a geometrically nonlinear lattice in the plane which, in the limit of low-energy, behaved as a "sonic 40 vacuum" (i.e., it had zero linearized speed of sound). Non-reciprocal dynamics was in the form of targeted energy transfers from large to small length scales, whereas non-reciprocal acoustics involved irreversible nonlinear wave interactions between predominantly transverse propagating localized pulses and axial (sonic) traveling waves.

To date there is no general theoretical framework for nonlinear non-reciprocity in nonlinear elastic systems. Accordingly, the main goal of the present work is to develop a theoretical framework for non-reciprocity in nonlinear elastodynamics that is applicable to a broad class of nonlinear time-invariant systems. Focusing on elastic systems with smooth stiffness nonlinearities, we develop conditions for non-reciprocal response based on multi-harmonic Volterra series expansions and asymptotic analysis. Apart from formulating necessary and sufficient conditions for nonlinear acoustic and dynamic non-reciprocity, we aim to develop quantitative non-reciprocity measures based on which optimization procedures can be developed, and, in the process, to reveal the passively self-tuning nature of non-reciprocal nonlinear elastic systems. Then, we provide an example that highlights the theoretical results and conclude by providing a synopsis of the main findings of this work. 


\section{Asymptotic analysis based on Volterra series (VS) expansions}

Our task to develop a general theoretical framework for acoustic non-reciprocity

60 extended nonlinearities is based on multi-harmonic Volterra series (VS) expansions. The use of VS in nonlinear systems is not new [19]22], and it enables the construction of higher-order transfer functions and spectra 23, 24, to generalize the linear concept of single-frequency transfer function. In particular, tion possess sufficiently smooth nonlinearities. In the following analysis we will assume that the developed VS representations are convergent. This clearly holds for the systems with polynomial-type stiffness nonlinearities (e.g., cubic stiffness terms) considered herein, whereas non-smooth nonlinearities such as clearances,

We consider the general one-dimensional (1D), undamped elastic waveguide governed by the following nonlinear partial differential equation,

$$
u_{t t}(x, t)=\mathcal{L}[u(x, t)]+\mathcal{N}[u(x, t)]+\vartheta f(x, t), \quad(x, t) \in \Omega \times \mathbb{R}^{+}
$$

defined over the spatial domain $\Omega$, and subject to the homogeneous LTI boundary conditions $\mathcal{B}[u]=0$ on $\partial \Omega$. In $(1)$, the quantity $u(x, t)$ is a scalar response (displacement) field with $x$ and $t$ being the spatial and temporal variables, respectively, $\mathcal{L}[\cdot]$ a self-adjoint LTI operator, $\mathcal{N}[\cdot]$ an essentially nonlinear (i.e., non-linearizable) time-invariant operator, $f(x, t)$ the external forcing function defined in $\Omega$ for $t \geq 0$, and $\vartheta$ is a small parameter $(|\vartheta| \ll 1)$ which will be used in the following asymptotic analysis. Linear viscous damping can be included in this formulation but we omit it in order to more clearly study the connection 
between stiffness nonlinearity and non-reciprocity; moreover, extension of the analysis to higher dimensions is straightforward from a mathematical point of view, but we refer to our comments in $\S 4$ for the new non-reciprocal physical wave phenomena that are expected in higher dimensions. We aim to study conditions for acoustic non-reciprocity in system (1) by applying a punctual excitation at a given position and studying the symmetry (reciprocity) properties of the response with respect to the points of excitation and measurement. To this end, we assume that the nonlinear operator in Eq. (1) is sufficiently smooth so that it may be Taylor-expanded in the form $\mathcal{N}[u]=a_{2} u^{2}+a_{3} u^{3}+\mathcal{O}\left(u^{4}\right)$, $a_{i} \in \mathbb{R}$.

To proceed, we consider an impulsive excitation applied at position $x=x_{0}$ and express the forcing function as $f\left(x, t ; x_{0}\right)=\delta\left(x-x_{0}\right) \phi(t)$, with the system initially at rest. Assuming small responses (so that we confine our attention to the weakly nonlinear regime), expressing the resulting response in power series in terms of the small parameter, $u=\sum_{m=1}^{N} \vartheta^{m} u_{m}$, substituting into Eq. (1), and matching terms of same powers of $\vartheta$, we obtain a hierarchy of sub-problems at different orders of approximation. At the leading order of approximation we obtain,

$$
\mathcal{O}(\vartheta): \quad u_{1, t t}-\mathcal{L}\left[u_{1}\right]=\delta\left(x-x_{0}\right) \phi(t) \quad \Longrightarrow \quad u_{1}\left(x, t ; x_{0}\right)=\mathcal{G} * f
$$

where $\mathcal{G}\left(x, t ; x_{0}\right) \equiv \mathcal{G}^{x, x_{0}}(t)$ denotes the Green's function of the operator $\left(\partial^{2} / \partial t^{2}-\right.$ $\mathcal{L})[\cdot]$ in $\Omega \times \mathbb{R}^{+}$for a unit impulse applied at $x=x_{0}$, at $t=0$. Moreover, the convolution operation over space and time is denoted by $*$ and defined as:

$\mathcal{G} * f \equiv(\mathcal{G} * f)\left(x, t ; x_{0}\right)=\int_{0}^{t} \int_{\Omega} \mathcal{G}^{x, \xi}(\tau) f\left(\xi, t-\tau ; x_{0}\right) \mathrm{d} \xi \mathrm{d} \tau=\int_{0}^{t} \mathcal{G}^{x, x_{0}}(\tau) \phi(t-\tau) \mathrm{d} \tau$

This compact notation will be used throughout the following analysis. The firstorder approximation $u_{1}\left(x, t ; x_{0}\right)$ is reciprocal since the Green's function $\mathcal{G}^{x, \xi}(t)$ is symmetric due to the self-adjointness of the operator $\left(\partial^{2} / \partial t^{2}-\mathcal{L}\right)[\cdot]$. Consid- 
ering the next orders of approximation we obtain the following sub-problems,

$$
\begin{array}{cc}
\mathcal{O}\left(\vartheta^{2}\right): & u_{2, t t}-\mathcal{L}\left[u_{2}\right]=a_{2} u_{1}^{2} \quad \Longrightarrow \quad u_{2}\left(x, t ; x_{0}\right)=a_{2} \mathcal{G} * u_{1}^{2} \\
\mathcal{O}\left(\vartheta^{3}\right): & u_{3, t t}-\mathcal{L}\left[u_{3}\right]=2 a_{2} u_{1} u_{2}+a_{3} u_{1}^{3} \\
\Longrightarrow u_{3}\left(x, t ; x_{0}\right)=a_{3} \mathcal{G} * u_{1}^{3}+2 a_{2} \mathcal{G} *\left(u_{1} u_{2}\right)
\end{array}
$$

whereas higher-order terms can be computed similarly. If $\vartheta$ is sufficiently small and the nonlinearity sufficiently smooth, the Volterra series converges [25, 26]. Considering the mathematical structure of the higher-order responses we note that in general, the higher-order approximations (3a), (3b), ... are not necessarily reciprocal due to the non-commutative nature of the variables $x$ and $x_{0}$ in the nested products of the powers of the Green's functions. In fact, as shown in the following analysis, the symmetry of the higher-order approximations [and, hence, the non-reciprocity of the original system (1) in space] depends on factors such as the boundary conditions, the possible symmetries between the excitation and measurement positions, and the type and spatial distribution of the nonlinearity. These conditions, however, can be rigorously studied by analyzing the structure of the VS terms $(2)-(3 \mathrm{a}, 3 \mathrm{~b}, \ldots)$, so a direct relation between nonlinearity and non-reciprocity can be established. To study analytically in more detail the non-reciprocity in Eq. (1) and highlight its dependence on nonlinearity, the boundary conditions and the domain of definition of the system, we will consider the specific example of the wave equation with cubic stiffness nonlinearity.

Before proceeding with the analysis, we note that the same VS-based approach can be applied also to the case of linear time-varying (LTV) systems (since time-variation of system properties is an alternative way to break acoustic reciprocity). This is performed by replacing the LTI operator in Eq. (1) by the LTV operator $\mathcal{L}_{1}[u, t]$ (depending explicitly on time), setting $\mathcal{N}[u] \equiv 0$, and following the previous asymptotic expansion in terms of the small parameter $\vartheta$. This, however, is not pursued further in the proposed work, since its exclusive focus is on nonlinear systems.

To study in more detail the efficacy of the proposed methodology to relate 
nonlinearity with non-reciprocity in the elastodynamic system (1), we restrict the analysis by considering the case of cubic stiffness nonlinearity, i.e., $\mathcal{N}[u] \equiv u^{3}$ in Eq. (1), and by assuming that $a_{2}=0$ and $a_{i}=0$ for $i \geq 4$ in the previous Taylor-series expansion of $\mathcal{N}[u]$. We note at this point that, whereas our formulation, analysis, and results extend to other types of stiffness or damping nonlinearities (e.g., quadratic ones), the following exposition centers exclusively around cubic stiffness nonlinearities since these typically arise in acoustic waveguides supported by linear springs but possessing geometric nonlinear effects see [18]. It follows that system (1) becomes

$$
u_{t t}=\mathcal{L}[u]+u^{3}+\vartheta f, \quad f\left(x, t ; x_{0}\right)=\delta\left(x-x_{0}\right) \phi(t) .
$$

Next, we consider two different types of boundary conditions. In particular, we consider the case of an unbounded elastic continuum, where Eq. (4) is defined on the infinite spatial domain $\Omega=\{x \in \mathbb{R},-\infty \leq x \leq+\infty\}$ with $|u|$ integrable; this we designate as Case I. Alternatively, we consider Eq. (4) on the finite domain $\Omega=\{x \in \mathbb{R}, 0 \leq x \leq 2 \pi\}$ with boundary conditions $u=0$ on $\partial \Omega=\{x \in \mathbb{R}, x=0, x=2 \pi\}$, and this we designate as Case II. Moreover, we set $\mathcal{L}[u]=u_{x x}$ so that system (4) becomes the $1 \mathrm{D}$ nonlinear Klein-Gordon system 2931 .

Under these assumptions, applying the previous asymptotic expansions renders the response of Eq. (4) in the form

$$
u\left(x, t ; x_{0}\right)=\vartheta \mathcal{G} * f+\vartheta^{3} \mathcal{G} *(\mathcal{G} * f)^{3}+\vartheta^{5}\left[3 \mathcal{G} *\left\{[\mathcal{G} * f]^{2}\left[\mathcal{G} *(\mathcal{G} * f)^{3}\right]\right\}\right]+\mathcal{O}\left(\vartheta^{7}\right)
$$

where the Green's function assumes different forms for the infinite and finite systems as follows:

Case I: System of infinite spatial extent

$$
\mathcal{G}^{x, \xi}(t)=(1 / 2) H(t-|x-\xi|), \quad \Omega=\{x \in \mathbb{R},-\infty \leq x \leq+\infty\}
$$


Case II: Finite system

$$
\begin{gathered}
\mathcal{G}^{x, \xi}(t)=\frac{1}{2} \sum_{n=-\infty}^{+\infty}[\underbrace{H(t-|x-\xi-2 \pi n|)}_{\text {Source at } x=2 \pi n+\xi}-\underbrace{H(t-|x+\xi-2 \pi n|)}_{\text {Sink at } x=2 \pi n-\xi}] \\
=\sum_{k=1}^{\infty} \frac{2}{k \pi} \sin \frac{k t}{2} \sin \frac{k x}{2} \sin \frac{k \xi}{2}, \\
\Omega=\{x \in \mathbb{R}, 0 \leq x \leq 2 \pi\} .
\end{gathered}
$$

In the above, $H(\cdot)$ is the Heaviside function. The differences between the mathematical structures of the Green's functions (6a) and (6b) have important implications on non-reciprocity.

\subsection{Case I: Boundless nonlinear system in the infinite spatial domain}

Considering first Case I, and noting that the leading-order term in Eq. 5 is reciprocal due to the symmetry of the Green's function, we examine the nextorder term and show that the nonlinear system (4) on the infinite domain $\Omega$ with the Green's function $\mathcal{G}^{x, \xi}(t)$ given by $(6 a)$ is reciprocal in space. To this end, we can show that in this case the terms $(\mathcal{G} * f)^{p}$, where $p=2,3, \ldots$, are reciprocal in space, and so are their convolutions with the Green's function, $\mathcal{G} *(\mathcal{G} * f)^{p}$. For the latter case (the former case is proven using similar arguments) the proof is as follows:

$$
\begin{aligned}
& \mathcal{G} *(\mathcal{G} * f)^{p}\left(x, t ; x_{0}\right) \\
= & \int_{0}^{t} \int_{-\infty}^{+\infty} \mathcal{G}^{x, \xi}(\tau)\left[\int_{0}^{t-\tau} \mathcal{G}^{\xi, x_{0}}(t-\tau-\sigma) \phi(\sigma) \mathrm{d} \sigma\right]^{p} \mathrm{~d} \xi \mathrm{d} \tau \\
= & \int_{0}^{t} \int_{-\infty}^{+\infty} \frac{1}{2} H(\tau-|x-\xi|)\left[\int_{0}^{t-\tau} \frac{1}{2} H\left(t-\tau-\sigma-\left|\xi-x_{0}\right|\right) \phi(\sigma) \mathrm{d} \sigma\right]^{p} \mathrm{~d} \xi \mathrm{d} \tau \\
= & \int_{0}^{t} \int_{-\infty}^{+\infty} \frac{1}{2} H\left(\tau-\left|\eta-x_{0}\right|\right)\left[\int_{0}^{t-\tau} \frac{1}{2} H(t-\tau-\sigma-|x-\eta|) \phi(\sigma) \mathrm{d} \sigma\right]^{p} \mathrm{~d} \eta \mathrm{d} \tau \\
= & \int_{0}^{t} \int_{-\infty}^{+\infty} \frac{1}{2} H\left(\tau-\left|x_{0}-\eta\right|\right)\left[\int_{0}^{t-\tau} \frac{1}{2} H(t-\tau-\sigma-|\eta-x|) \phi(\sigma) \mathrm{d} \sigma\right]^{p} \mathrm{~d} \eta \mathrm{d} \tau \\
= & \int_{0}^{t} \int_{-\infty}^{+\infty} \mathcal{G}^{x_{0}, \eta}(\tau)\left[\int_{0}^{t-\tau} \mathcal{G}^{\eta, x}(t-\tau-\sigma) \phi(\sigma) \mathrm{d} \sigma\right]^{p} \mathrm{~d} \eta \mathrm{d} \tau \\
= & \mathcal{G} *(\mathcal{G} * f)^{p}\left(x_{0}, t ; x\right) .
\end{aligned}
$$


In the proof above, the change of variables $\eta=x_{0}+x-\xi$ was introduced. It follows that by setting $p=3$ we prove that the $\mathcal{O}\left(\vartheta^{3}\right)$ leading-order nonlinear approximation in Eq. (5) is reciprocal. We can show similarly that, for any function $F(\cdot)$ such that $\mathcal{G} * F(\mathcal{G} * f)$ exists and is bounded for all $x, x_{0}$ and $t$, the combined function $\mathcal{G} * F(\mathcal{G} * f)$ is reciprocal in space on the infinite domain $\Omega$. Based on these results, we prove that products of the form $[\mathcal{G} * f]^{q}\left[\mathcal{G} *(\mathcal{G} * f)^{p}\right]$ are reciprocal in space as well, which, in turn proves that the $\mathcal{O}\left(\vartheta^{5}\right)$ term and the higher-order terms of the asymptotic expansion (5) are reciprocal in space. Combining the previous results, we prove that the response (5) for Case I is reciprocal, so the nonlinear system (4) defined in the infinite domain (Case I) does not violate reciprocity in space. This result proves that nonlinearity does not necessarily imply non-reciprocity, and additional conditions should be met for non-reciprocal response. This is proved in the following analysis for Case II.

\subsection{Case II: Finite nonlinear system}

Considering now Case II of the finite nonlinear system (4) with $\mathcal{L}[u]=u_{x x}$ and Green's function defined by (6b), we will prove that, provided that $x=$ $2 \pi-x_{0}$, terms of the form $(\mathcal{G} * f)^{p}$ and $\mathcal{G} *(\mathcal{G} * f)^{p}$, where $p=2,3, \ldots$, are reciprocal in $\Omega$. This is shown as follows:

$$
\begin{aligned}
& \mathcal{G} *(\mathcal{G} * f)^{p}\left(x, t ; x_{0}\right)=\int_{0}^{t} \int_{0}^{2 \pi}\left[\sum_{k=1}^{\infty} \frac{2}{k \pi} \sin \frac{k \tau}{2} \sin \frac{k x}{2} \sin \frac{k \xi}{2}\right] \\
& \times\left[\int_{0}^{t-\tau} \sum_{m=1}^{\infty} \frac{2}{m \pi} \sin \frac{m(t-\tau-\sigma)}{2} \sin \frac{m \xi}{2} \sin \frac{m x_{0}}{2} \phi(\sigma) \mathrm{d} \sigma\right]^{p} \mathrm{~d} \xi \mathrm{d} \tau .
\end{aligned}
$$

Assuming that $x=2 \pi-x_{0}$, the above expression yields

$$
\begin{gathered}
\mathcal{G} *(\mathcal{G} * f)^{p}\left(x=2 \pi-x_{0}, t ; x_{0}\right)=\int_{0}^{t} \int_{0}^{2 \pi}\left[\sum_{k=1}^{\infty} \frac{2}{k \pi}(-1)^{k+1} \sin \frac{k \tau}{2} \sin \frac{k x_{0}}{2} \sin \frac{k \xi}{2}\right] \\
\times\left[\int_{0}^{t-\tau} \sum_{m=1}^{\infty} \frac{2}{m \pi}(-1)^{m+1} \sin \frac{m(t-\tau-\sigma)}{2} \sin \frac{m \xi}{2} \sin \frac{m x}{2} \phi(\sigma) \mathrm{d} \sigma\right]^{p} \mathrm{~d} \xi \mathrm{d} \tau .
\end{gathered}
$$


Finally, introducing the change of variables $\eta=2 \pi-\xi$ in Eq. $8 \mathrm{~b}$ yields

$$
\begin{gathered}
\mathcal{G} *(\mathcal{G} * f)^{p}\left(x=2 \pi-x_{0}, t ; x_{0}\right)=\int_{0}^{t} \int_{0}^{2 \pi}\left[\sum_{k=1}^{\infty} \frac{2}{k \pi}(-1)^{2(k+1)} \sin \frac{k \tau}{2} \sin \frac{k x_{0}}{2} \sin \frac{k \eta}{2}\right] \\
\times\left[\int_{0}^{t-\tau} \sum_{m=1}^{\infty} \frac{2}{m \pi}(-1)^{2(m+1)} \sin \frac{m(t-\tau-\sigma)}{2} \sin \frac{m \eta}{2} \sin \frac{m x}{2} \phi(\sigma) \mathrm{d} \sigma\right]^{p} \mathrm{~d} \eta \mathrm{d} \tau \\
=\int_{0}^{t} \int_{0}^{2 \pi}\left[\sum_{k=1}^{\infty} \frac{2}{k \pi} \sin \frac{k \tau}{2} \sin \frac{k x_{0}}{2} \sin \frac{k \eta}{2}\right] \\
\times\left[\int_{0}^{t-\tau} \sum_{m=1}^{\infty} \frac{2}{m \pi} \sin \frac{m(t-\tau-\sigma)}{2} \sin \frac{m \eta}{2} \sin \frac{m x}{2} \phi(\sigma) \mathrm{d} \sigma\right]^{p} \mathrm{~d} \eta \mathrm{d} \tau \\
=\int_{0}^{t} \int_{0}^{2 \pi} \mathcal{G}^{x_{0}, \eta}(\tau)\left[\int_{0}^{t-\tau} \mathcal{G}^{\eta, x}(t-\tau-\sigma) \phi(\sigma) \mathrm{d} \sigma\right]^{p} \mathrm{~d} \eta \mathrm{d} \tau \\
=\mathcal{G} *(\mathcal{G} * f)^{p}\left(x_{0}, t ; x=2 \pi-x_{0}\right) .
\end{gathered}
$$

This proves that in Case II, provided that $x=2 \pi-x_{0} \in \Omega$, the $\mathcal{O}\left(\vartheta^{3}\right)$ approximation in Eq. (5) is reciprocal in space. Using similar ideas any higherorder approximations can be shown to be reciprocal in this case, i.e., successive changes of variables of the form $\xi_{i}=2 \pi-\xi_{j}$ in the convolution operations in order to swap out $x$ and $x_{0}$. This proves that for $x=2 \pi-x_{0} \in \Omega$, the nonlinear system (4) defined in the finite domain (Case II) does not violate reciprocity in space. However, any small perturbation $x=2 \pi-x_{0}+\varepsilon \in \Omega$ with $0<\varepsilon \ll 1$, i.e., any small perturbation in the symmetric choice of $x$ and $x_{0}$ in $\Omega$, would lead to non-reciprocity in space for Case II. This is proven following the previous analytical derivation and substituting $x=2 \pi-x_{0}+\varepsilon$ in Eq. 8a to yield

$$
\begin{aligned}
\mathcal{G} *(\mathcal{G} * f)^{p}\left(x=2 \pi-x_{0}+\varepsilon, t ; x_{0}\right) \\
=\int_{0}^{t} \int_{0}^{2 \pi}\left\{\sum_{k=1}^{\infty}\left[\frac{2}{k \pi} \sin \frac{k x_{0}}{2}+\frac{\varepsilon}{\pi} \cos \frac{k x_{0}}{2}+\mathcal{O}\left(\varepsilon^{2}\right)\right](-1)^{k+1} \sin \frac{k \tau}{2} \sin \frac{k \xi}{2}\right\} \\
\times\left\{\int_{0}^{t-\tau} \sum_{m=1}^{\infty}\left[\frac{2}{m \pi} \sin \frac{m x}{2}+\frac{\varepsilon}{\pi} \cos \frac{m x}{2}+\mathcal{O}\left(\varepsilon^{2}\right)\right]\right. \\
\left.\times(-1)^{m+1} \sin \frac{m(t-\tau-\sigma)}{2} \sin \frac{m \xi}{2} \phi(\sigma) \mathrm{d} \sigma\right\}^{p} \mathrm{~d} \xi \mathrm{d} \tau
\end{aligned}
$$


Letting $\eta=2 \pi-\xi$ in (9a) yields

$$
\begin{aligned}
\mathcal{G} *(\mathcal{G} * f)^{p}\left(x=2 \pi-x_{0}+\varepsilon, t ; x_{0}\right) \\
=\int_{0}^{t} \int_{0}^{2 \pi}\left\{\sum_{k=1}^{\infty}\left[\frac{2}{k \pi} \sin \frac{k x_{0}}{2}+\frac{\varepsilon}{\pi} \cos \frac{k x_{0}}{2}+\mathcal{O}\left(\varepsilon^{2}\right)\right] \sin \frac{k \tau}{2} \sin \frac{k \eta}{2}\right\} \\
\times\left\{\int_{0}^{t-\tau} \sum_{m=1}^{\infty}\left[\frac{2}{m \pi} \sin \frac{m x}{2}+\frac{\varepsilon}{\pi} \cos \frac{m x}{2}+\mathcal{O}\left(\varepsilon^{2}\right)\right]\right. \\
\left.\times \sin \frac{m(t-\tau-\sigma)}{2} \sin \frac{m \eta}{2} \phi(\sigma) \mathrm{d} \sigma\right\}^{p} \mathrm{~d} \eta \mathrm{d} \tau .
\end{aligned}
$$

We recall at this point the definition $6 \mathrm{~b})$ of the Green's function for Case II, yielding:

$$
\begin{aligned}
\mathcal{G}^{x, \eta}(t) & =\sum_{k=1}^{\infty} \frac{2}{k \pi} \sin \frac{k t}{2} \sin \frac{k x}{2} \sin \frac{k \eta}{2} \\
\frac{\partial \mathcal{G}^{x, \eta}(t)}{\partial x} & \equiv \mathcal{G}_{x}^{x, \eta}(t)=\sum_{k=1}^{\infty} \frac{1}{\pi} \sin \frac{k t}{2} \cos \frac{k x}{2} \sin \frac{k \eta}{2} .
\end{aligned}
$$

We note that the series $\mathcal{G}_{x}^{x, \eta}(t)$ diverges in the classical sense but converges in the sense of the theory of distributions [32. We note also that, whereas $\mathcal{G}^{x, \eta}(t)$ is reciprocal in $\Omega$ [since $\mathcal{G}^{x, \eta}(t)=\mathcal{G}^{\eta, x}(t)$ for $x, \eta \in \Omega=\{y \in \mathbb{R}, 0 \leq y \leq 2 \pi\}$ ], a simple computation shows that $\mathcal{G}_{x}^{x, \eta}(t)$ is non-reciprocal. Hence, $(9 \mathrm{~b})$ can be expressed as follows:

$$
\begin{aligned}
& \mathcal{G} *(\mathcal{G} * f)^{p}\left(x=2 \pi-x_{0}+\varepsilon, t ; x_{0}\right)=\int_{0}^{t} \int_{0}^{2 \pi}\left\{\mathcal{G}^{x_{0}, \eta}(\tau)+\varepsilon \mathcal{G}_{x}^{x_{0}, \eta}(\tau)+\mathcal{O}\left(\varepsilon^{2}\right)\right\} \\
& \times\left\{\int_{0}^{t-\tau}\left[\mathcal{G}^{x, \eta}(t-\tau-\sigma)+\varepsilon \mathcal{G}_{x}^{x, \eta}(t-\tau-\sigma)+\mathcal{O}\left(\varepsilon^{2}\right)\right] \phi(\sigma) \mathrm{d} \sigma\right\}^{p} \mathrm{~d} \eta \mathrm{d} \tau .
\end{aligned}
$$

If we assume for the moment that $p=1$ (no nonlinearity) in Eq. 11, and collect powers of $\varepsilon$, we obtain

$$
\begin{gathered}
\mathcal{G} *(\mathcal{G} * f)\left(x=2 \pi-x_{0}+\varepsilon, t ; x_{0}\right) \\
=\int_{0}^{t} \int_{0}^{2 \pi} \mathcal{G}^{x_{0}, \eta}(\tau)\left[\int_{0}^{t-\tau} \mathcal{G}^{\eta, x}(t-\tau-\sigma) \phi(\sigma) \mathrm{d} \sigma\right] \mathrm{d} \eta \mathrm{d} \tau \\
+\varepsilon\left\{\int _ { 0 } ^ { t } \int _ { 0 } ^ { 2 \pi } \left[\mathcal{G}_{x}^{x_{0}, \eta}(\tau) \int_{0}^{t-\tau} \mathcal{G}^{\eta, x}(t-\tau-\sigma) \phi(\sigma) \mathrm{d} \sigma\right.\right. \\
\left.\left.+\mathcal{G}^{x_{0}, \eta}(\tau) \int_{0}^{t-\tau} \mathcal{G}_{x}^{x, \eta}(t-\tau-\sigma) \phi(\sigma) \mathrm{d} \sigma\right] \mathrm{d} \eta \mathrm{d} \tau\right\}+\mathcal{O}\left(\varepsilon^{2}\right),
\end{gathered}
$$


which is reciprocal for all powers of $\varepsilon$ by virtue of the commutativity of the convolution in time and space symmetry of $G$. Reciprocity is expected in this case since $\mathcal{G} *(\mathcal{G} * f)$ is reciprocal for any choice of $x, x_{0} \in \Omega$. In particular, we notice how the two nonreciprocal cross-convolutions between $\mathcal{G}_{x}$ and $\mathcal{G}$ add up to yield a reciprocal term at order $\varepsilon$. In what follows, we show how the nonlinearity breaks this pattern, and therefore destroys reciprocity.

Considering again Eq. (11) with $p \geq 2$, we again collect powers of $\varepsilon$ and derive the expression

$$
\begin{gathered}
\mathcal{G} *(\mathcal{G} * f)^{p}\left(x=2 \pi-x_{0}+\varepsilon, t ; x_{0}\right)= \\
\int_{0}^{t} \int_{0}^{2 \pi} \mathcal{G}^{x_{0}, \eta}(\tau)\left[\int_{0}^{t-\tau} \mathcal{G}^{\eta, x}(t-\tau-\sigma) \phi(\sigma) \mathrm{d} \sigma\right]^{p} \mathrm{~d} \eta \mathrm{d} \tau \\
+\varepsilon \\
\left\{\int_{0}^{t} \int_{0}^{2 \pi}\left[\int_{0}^{t-\tau} \mathcal{G}^{\eta, x}(t-\tau-\sigma) \phi(\sigma) \mathrm{d} \sigma\right]^{p-1}\right. \\
\times\left[\mathcal{G}_{x}^{x_{0}, \eta}(\tau) \int_{0}^{t-\tau} \mathcal{G}^{\eta, x}(t-\tau-\sigma) \phi(\sigma) \mathrm{d} \sigma\right. \\
\left.\left.+p \mathcal{G}^{x_{0}, \eta}(\tau) \int_{0}^{t-\tau} \mathcal{G}_{x}^{x, \eta}(t-\tau-\sigma) \phi(\sigma) \mathrm{d} \sigma\right] \mathrm{d} \eta \mathrm{d} \tau\right\}+\mathcal{O}\left(\varepsilon^{2}\right) .
\end{gathered}
$$

The leading-order term in Eq. 13 was shown to be reciprocal earlier (it corresponds to the symmetric case $x=2 \pi-x_{0} \in \Omega$ for which $\left.\varepsilon=0\right)$. The $\mathcal{O}(\varepsilon)$ term in Eq. (13), however, is non-reciprocal for $p>1$. Indeed, only if $p=1$ does the term raised to the $(p-1)$ power out-front become equal to unity, and we recover Eq. (12), which is reciprocal. From the previous derivations it is proven that if, $x=2 \pi-x_{0}+\varepsilon \in \Omega$ with $0<\varepsilon \ll 1$, then $\mathcal{G} *(\mathcal{G} * f)^{p}\left(x, t ; x_{0}\right) \neq \mathcal{G} *(\mathcal{G} * f)^{p}\left(x_{0}, t ; x\right)$, i.e., that any asymmetric choice of $x$ and $x_{0}$ in $\Omega$ breaks spatial reciprocity in Case II.

It follows that the symmetry (or lack thereof) of the boundary conditions with respect to sensing positions in a finite acoustic waveguide plays an important role in the nonlinear case, but not in the linear one. The reason is the amplitude (or, equivalently, energy) dependence of the dispersion relationship in the nonlinear acoustic medium, and the absence of a similar amplitude dependence in the linear case (in the linear case the dispersion relationship is 
between the frequency and the wavenumber or phase speed). As a result, asymmetrically placed boundary conditions in a nonlinear acoustic waveguide affects the speed and phase of right- and left-going waves in the waveguide as these waves encounter and are reflected by the boundaries. In turn, reflections from asymmetrically placed boundaries affect the positive or negative interference patterns of left- and right-going waves at the sensing positions in the nonlinear acoustic medium, since these patterns are significantly influenced by the distance of the sensing positions from the boundaries, as well as the amplitudes of the propagating waves as they are reflected at the boundaries, and/or are dispersed by the nonlinear medium. It follows that in the nonlinear acoustic waveguide, the responses at the sensing positions are critically affected by the relative placements of the sensing positions with respect to the boundaries, whereas there are no such effects in boundless nonlinear waveguides. This generates nonlinear acoustic non-reciprocity, as shown formally in this section. In the corresponding linear waveguides, there is no amplitude dependence of the dispersion relation, so the aforementioned wave interference patterns are not affected by the asymmetry of the boundaries, and linear acoustic reciprocity is a direct result of the symmetry of the Green's function, which, in turn, is the result of the self-adjointness of the governing LTI differential operator.

\subsection{Non-reciprocity quantification measures}

The previous analytical findings show that nonlinearity is neither necessary nor sufficient for breaking reciprocity in the 1D nonlinear system (4). Rather, non-reciprocity in this nonlinear system depends on the boundary conditions and on the choice of the spatial points where this non-reciprocity is tested. More specifically, based on our findings we may conjecture that 1D nonlinear elastic systems defined over boundless domains (e.g., Case I studied herein) are in general reciprocal; whereas finite elastodynamic systems (e.g., Case II considered here) may or may not be reciprocal, depending on certain symmetry conditions satisfied by the boundary conditions and the spatial points where reciprocity is tested. Generalization of the mathematical analysis to higher 
dimensional elastodynamics is straightforward.

Considering again systems (1) or (4), the non-reciprocity of the nonlinear response can be defined through appropriate quantification measures in order to perform non-reciprocity quantification. A possible non-reciprocity quantification measure can be defined by separating the reciprocal and non-reciprocal parts of a measured time series response according to the following expressions,

$$
\begin{aligned}
& \rho_{\mathrm{r}}\left(t ; x, x_{0}\right)=\left|u\left(x_{0}, t ; x\right)+u\left(x, t ; x_{0}\right)\right| / 2 \quad \text { (Reciprocal part) } \\
& \rho_{\mathrm{nr}}\left(t ; x, x_{0}\right)=\left|u\left(x_{0}, t ; x\right)-u\left(x, t ; x_{0}\right)\right| / 2 \quad \text { (Non-reciprocal part) }
\end{aligned}
$$

where in the notation for the response $u(y, t ; z), y$ and $z$ denote the measurement and excitation locations, respectively. We may eliminate the time dependencies from the non-reciprocity measures by introducing the following two alternative expressions for two specific interchangeable measurement/excitation positions:

$$
\begin{aligned}
& M_{1}\left(x, x_{0}\right)=\operatorname{RMS}\left\{\left|u\left(x_{0}, t ; x\right)-u\left(x, t ; x_{0}\right)\right|\right\} \\
& M_{2}\left(x, x_{0}\right)=\frac{1}{T} \int_{0}^{T}\left|u\left(x_{0}, \tau ; x\right)-u\left(x, \tau ; x_{0}\right)\right| \mathrm{d} \tau .
\end{aligned}
$$

Based on these non-reciprocity quantification measures, optimization can be performed to study the effects of the type and distribution of the nonlinearity, the energy level, the types of boundary conditions, the possible symmetries, and of other factors on non-reciprocity. To this end, numerical optimization tools previously used in the optimal design and system identification of strongly nonlinear structures [33, 34] can be adapted to refine parameter values toward this end, using the non-reciprocity measure as an objective function. Experience has shown both gradient-based and genetic-algorithm optimizers to be effective for this work. Such an approach can lead to a better physical understanding of non-reciprocity in a broad class of systems, and can provide important insight that will facilitate its effective and robust implementation.

In addition, the outlined perturbation methodology is especially suitable for studying the effects of nonlinear inhomogeneities on non-reciprocity. For example, assuming that the Green's function $\mathcal{G}^{x, \xi}(t)$ has a discrete spectrum (e.g., 
Case II), the response of (1) or (4) can be projected onto the eigenbasis of the operator $\left(\partial^{2} / \partial t^{2}-\mathcal{L}\right)[\cdot]$, leading to reduced-order modeling. Such an order reduction can shed further physical insight, as it can enable the investigation of the role of irreversible multi-scale nonlinear targeted energy transfers in nonreciprocity. In that context, the influence of the spectral composition of the Green's functions on acoustic non-reciprocity would be worth examining. Especially in the case of nonlinear phononic structures [35] the influence of their energy-dependent pass- and stop-bands on non-reciprocity would be an interesting topic of further study. Finally, it is straightforward to extend the previous VS-based asymptotic framework (which was focused on spatial non-reciprocity) to the study of time irreversibility of the nonlinear responses of (1) or (4). To address this task, we will need to introduce an extended Green's function of the form $\tilde{\mathcal{G}}(x, t ; \xi, \tau)$ subject to the combined spatio-temporal unit excitation

${ }_{225} \delta(x-\xi) \delta(t-\tau)$. Then, a similar VS analysis based on $\tilde{\mathcal{G}}$ can be performed to study time irreversibility.

\section{Numerical example}

A numerical application is provided with the system of Fig. 1 1 consisting of a linear finite non-dispersive string with (normalized) unit length, fixed boundary conditions and a strongly nonlinear local stiffness at position $x=x_{0}$. Assuming the nonlinear characteristic $f(u)=-\left(k_{1} u+k_{3} u^{3}\right)$ for the local stiffness, the nonlinear elastodynamics of this system is governed by the following normalized equation of motion and boundary conditions:

$$
u_{t t}=u_{x x}-\left(k_{1} u+k_{3} u^{3}\right) \delta\left(x-x_{0}\right), \quad u(0, t)=u(1, t)=0 .
$$

We consider the response of this system for a unit impulse applied at $t=$ $0^{+}$, at location $x=x_{\text {imp }}$, subject to zero initial conditions, $u\left(x, 0^{-}\right)=0$ and $u_{t}\left(x, 0^{-}\right)=0$. This amounts to the following initial conditions for the string immediately after the application of the impulse,

$$
u\left(x, 0^{+}\right)=0, \quad u_{t}\left(x, 0^{+}\right)=\exp \left[-\frac{\left(x-x_{\mathrm{imp}}\right)^{2}}{2 \sigma^{2}}\right]
$$


which is normalized according to $E_{0}=(1 / 2) \int_{0}^{1} u_{t}^{2}\left(x, 0^{+}\right) \mathrm{d} x$.

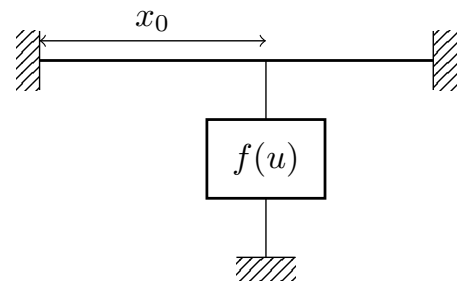

Figure 1: Finite string with strongly nonlinear stiffness.

To test reciprocity for this system we consider parameters $x_{0}=1 / 2, E_{0}=5$, 230 $\sigma=0.005, k_{1}=1$, and nonlinear stiffness coefficient in the range $10^{-2} \leq k_{3} \leq$ $10^{5}$. Moreover, the following two scenarios are considered, corresponding to the interchange of two forcing and measurement positions:

Scenario 1

$$
\begin{array}{lr}
\text { Forcing location: } & x_{\mathrm{imp}} \equiv x_{A}=0.3 \\
\text { Measurement location: } & x_{\text {meas }} \equiv x_{B}=0.4
\end{array}
$$

$\underline{\text { Scenario } 2}$

$$
\begin{array}{lr}
\text { Forcing location: } & x_{\mathrm{imp}} \equiv x_{A^{\prime}}=0.4 \\
\text { Measurement location: } & x_{\text {meas }} \equiv x_{B^{\prime}}=0.3
\end{array}
$$

In Fig. 2 we depict representative responses of this system for the linear case $\left(k_{3}=0\right)$ and a strongly nonlinear case $\left(k_{3}=10^{4}\right)$, from which the nonlinear non-reciprocity is clearly denoted in the latter case. In Fig. 3 we depict the time-independent non-reciprocity measures $M_{1}$ and $M_{2}$ defined by expressions (15a) and 15b), respectively. Both measures predict exact and negligible nonreciprocity for $k_{3}=0$ and $0<k_{3} \ll 1$, as expected. For increasing $k_{3}$, the non-reciprocity in this system becomes evident, reaching a maximum value at $240 k_{3, \max } \approx 10^{2}$ for both non-reciprocity measures. This leads to the interesting conclusion that there is a specific value of the nonlinear stiffness coefficient that 
maximizes non-reciprocity in this system. Physically, the point of maximum non-reciprocity denotes the point of transition of the nonlinear elastodynamics between two limiting linear systems, namely the system with linear stiffness attachment corresponding to $k_{3}=0$ and the system with rigid attachment (i.e., a fixed boundary condition) corresponding to $k_{3} \rightarrow \infty$. This indicates that for $0<k_{3}<k_{3, \max }$ the nonlinear dynamics could be regarded as perturbation of the linear limiting system with $k_{3}=0$, whereas for $k_{3 \text {, max }}<k_{3}<\infty$, as perturbation of the other linear limiting system with $k_{3} \rightarrow \infty$; viewed in that context, the transition point could be considered heuristically as the point of equidistance between the two aforementioned linear limiting systems, or equivalently, as the point of "maximum nonlinearity" in the elastodynamics.

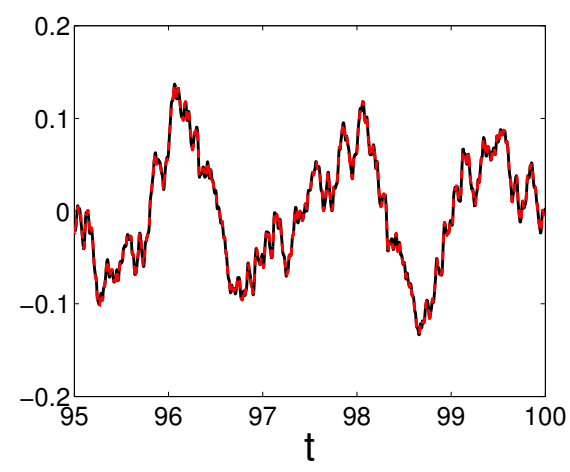

(a)

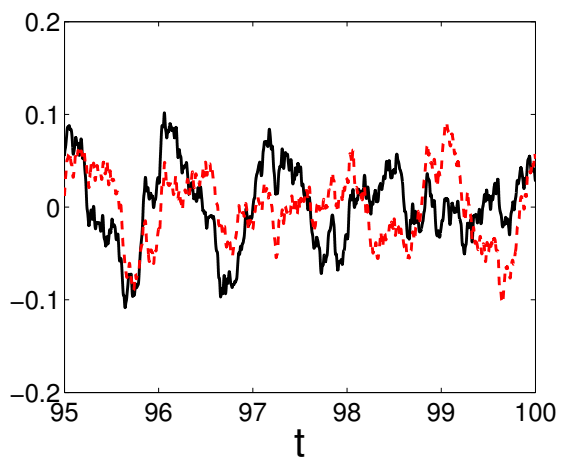

(b)

Figure 2: Responses $u\left(x_{B}, t ; x_{A}\right)$ (solid line) and $u\left(x_{B^{\prime}}, t ; x_{A^{\prime}}\right)$ (dashed line) for (a) $k_{3}=0$, and (b) $k_{3}=10^{4}$.

However, we note that the previous discussion is valid only for the specific pair of interchangeable excitation/measurement positions considered in the previous example (i.e., Scenarios 1 and 2), so for a more complete picture of nonreciprocity of the elastodynamics, one would need to vary continuously these positions to "scan" the entire length of the string. Nevertheless, this discussion highlights the potential of the presented theoretical analysis and the associated non-reciprocity measures for physically understanding and accurately quantify- 


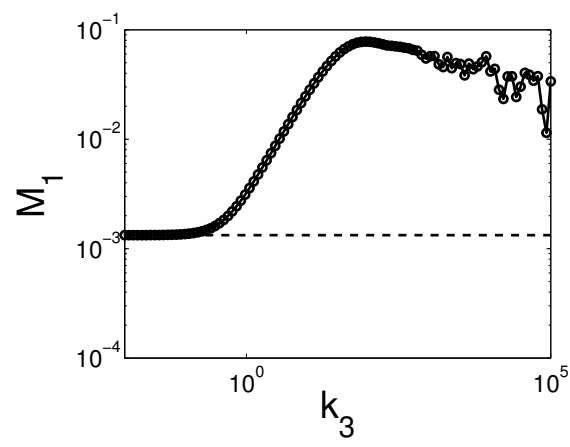

(a)

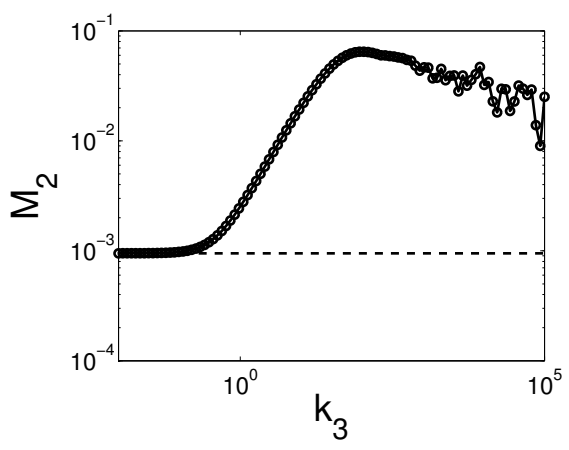

(b)

Figure 3: Non-reciprocity measures (a) $M_{1}$ and (b) $M_{2}$ for $k_{1}=1$ and $E_{0}=5$ for forcing/measurement Scenarios 1 and 2 .

ing the nonreciprocal elastodynamics of systems with local or distributed nonlinearities.

\section{Conclusions}

Using Volterra-series based asymptotic analysis, we studied non-reciprocity in space for a class of 1D continuous, time-invariant systems with stiffness nonlinearities. Nonlinearity is neither necessary nor sufficient for breaking reciprocity in this class of systems; rather, non-reciprocity depends on the boundary conditions, the symmetries (or disorder) of the governing linear and nonlinear operators, and the choice of the spatial points where non-reciprocity is tested. Generalization of the analysis to higher dimensional elastodynamics might be straightforward from a mathematical point of view since the developed asymptotic methodology can conveniently incorporate higher spatial dimensions (albeit with increased analytical complexity); however, from a physical point of view, the study of nonlinear acoustic non-reciprocity in higher dimensions is expected to reveal new interesting wave phenomena. For example, in higher dimensions, we expect nonlinear wave interactions that would couple waves propagating in different directions or corresponding to different modes of wave propagation (e.g., transverse versus axial - for an example of such nonlinear 
wave interactions in a two-dimensional geometrically nonlinear chain, we refer to [18]); possible nonlinear targeted energy transfers between different types of waves [18; the possibility of nonlinear complex wave modes (such wave modes appear already in higher dimensional linear acoustics). In fact, the interplay of nonlinearity and wave complexity, and their combined effects on acoustic nonreciprocity in higher dimensions is an interesting topic for further research; the effects of the boundary conditions on different types of waves, and their nonreciprocal influence on non-reciprocal wave propagation depending not only on the direction of wave propagation (as one of the reviewers of this work indicated), but also on the type of wave mode (e.g., shear versus axial waves), etc. Nevertheless, the developed Volterra-based asymptotic methodology of this work can be a basis for studying analytically such non-reciprocal wave phenomena in dimensions higher than one.

Furthermore, the developed non-reciprocity measures enable optimization studies leading to predictive design of systems with optimal non-reciprocity measures. In addition, they can provide better physical understanding on the nonlinear effects, helping one establish regimes of "maximum nonlinearity" or of near-linear reciprocal behavior. Finally, the presented analytical developments can be conveniently extended to understanding non-reciprocity in systems with time-varying properties (this is another possibility for breaking nonreciprocity), to studying time irreversibility in the dynamics, and to analyzing the non-reciprocal dynamics of elastic systems with combined local or extended stiffness and damping nonlinearities.

\section{References}

[1] A. D. Pierce, Acoustics: An introduction to its physical principles and applications, Acoustical Society of America, Woodbury, New York, 1981.

[2] J. D. Achenbach, Reciprocity in elastodynamics, Cambridge University Press, Cambridge, 2003. 
[3] R. Courant, D. Hilbert, Methods of mathematical physics, Wiley-VCH, New York, 1989.

[4] L. Onsager, Reciprocal relations in irreversible processes - I., Phys. Rev. 37 (1931) 405-426.

[5] L. Onsager, Reciprocal relations in irreversible processes - II., Phys. Rev. 38 (1931) 2265-2279.

[6] H. Casimir, On Onsager's principle of microscopic reversibility, Rev. of Mod. Phys. 17 (1945) 343-350.

[7] R. Fleury, D. Sounas, M. Haberman, A. Alù, Nonreciprocal acoustics, Acoustics Today 11 (2015) 14-21.

[8] R. Fleury, D. L. Sounas, C. F. Sieck, M. R. Haberman, A. Alù, Sound isolation and giant linear nonreciprocity in a compact acoustic circulator, Science 343 (2014) 516-519.

[9] S. A. Cummer, Selecting the direction of sound transmission, Science 343 (2014) 495-496.

[10] K. Tsakmakidis, L. Shen, S. Schulz, X. Zheng, J. Upham, X. Deng, H. Altug, A. Vakakis, R. Boyd, Breaking Lorentz reciprocity to overcome the time-bandwidth limit in physics and engineering, Science 356 (2017) 12601264 .

[11] R. Fleury, D. L. Sounas, A. Alù, Subwavelength ultrasonic circulator based on spatiotemporal modulation, Phys. Rev. B 91 (2015) 174306.

[12] B.-I. Popa, S. A. Cummer, Non-reciprocal and highly nonlinear active acoustic metamaterials, Nature Com. 5 (2014).

[13] M. Maldovan, Sound and heat revolutions in phononics, Nature 503 (2013) 209-217. 
[14] B. Liang, B. Yuan, J.-C. Cheng, Acoustic diode: Rectification of acoustic energy flux in one-dimensional systems, Phys. Rev. Lett. 103 (2009) 104301.

[15] B. Liang, X. Guo, J. Tu, D. Zhang, J. Cheng, An acoustic rectifier, Nature Mat. 9 (2010) 989-992.

335 [16] N. Boechler, G. Theocharis, C. Daraio, Bifurcation-based acoustic switching and rectification, Nature Mat. 10 (2011) 665-668.

[17] F. Li, P. Anzel, J. Yang, P. G. Kevrekidis, C. Daraio, Granular acoustic switches and logic elements, Nature Com. 5 (2014).

[18] Z. Zhang, I. Koroleva, L. Manevitch, L. Bergman, A. Vakakis, Nonreciprocal acoustics and dynamics in the in-plane oscillations of a geometrically nonlinear lattice, Phys. Rev. E 94 (2016) 032214.

[19] S. Gifford, G. Tomlinson, Recent advances in the application of functional series to non-linear structures, J. Sound Vib. 135 (1989) 289-317.

[20] I. Tawfiq, T. Vinh, Nonlinear behaviour of structures using the Volterra series - signal processing and testing methods, Nonl. Dyn. 37 (2004) 129149.

[21] A. Chatterjee, Parameter estimation of Duffing oscillator using Volterra series and multi-tone excitation, Int. J. Mech. Sciences 52 (2010) 17161722.

[22] L. Li, S. Billings, Analysis of nonlinear oscillators using Volterra series in the frequency domain, J. Sound Vib. 330 (2011) 337-355.

[23] S. Billings, K. Tsang, Spectral analysis for non-linear systems, Part II: Interpretation of non-linear frequency response functions, Mech. Sys. Signal Proc. 3 (1989) 341-359.

[24] D. M. Storer, Dynamic analysis of non-linear structures using higher order frequency response functions, Ph.D. thesis, University of Manchester, 1991. 
[25] S. Boyd, L. O. Chua, C. A. Desoer, Analytical foundations of Volterra series, IMA J. Math. Control Inf. 1 (1984) 243-282.

[26] S. Boyd, L. Chua, Fading memory and the problem of approximating nonlinear operators with Volterra series, IEEE Trans. Circ. Syst. 32 (1985) $1150-1161$.

[27] G. Tomlinson, G. Manson, G. Lee, A simple criterion for establishing an upper limit to the harmonic excitation level of the Duffing oscillator using the Volterra series, J. Sound Vib. 190 (1996) 751-762.

[28] A. Chatterjee, N. S. Vyas, Convergence analysis of Volterra series response of nonlinear systems subjected to harmonic excitation, J. Sound Vib. 236 (2000) 339-358.

[29] A. Weinstein, Periodic nonlinear waves on a half-line, Com. Math. Phys. 99 (1985) 385-388.

370 [30] V. Eleonsky, Problems of existence of nontopological solitons (breathers) for nonlinear Klein-Gordon equations, in: Asymptotics beyond All Orders, Springer, 1991, pp. 357-363.

[31] R. Zhdanov, Separation of variables in the nonlinear wave equation, J. Phys. A 27 (1994) L291-L297.

[32] R. Richtmyer, Principles of advanced mathematical physics, volume 1, Springer Verlag, Berlin and New York, 1978.

[33] S. Hubbard, Stability enhancement of a transonic wing using a passive nonlinear energy sink, Ph.D. thesis, University of Illinois at UrbanaChampaign, 2014.

${ }_{380}$ [34] N. E. Wierschem, S. A. Hubbard, J. Luo, L. A. Fahnestock, B. F. Spencer, D. M. McFarland, D. D. Quinn, A. F. Vakakis, L. A. Bergman, Response attenuation in a large-scale structure subjected to blast excitation utilizing 
a system of essentially nonlinear vibration absorbers, J. Sound Vib. 389 (2017) 52-72.

${ }_{385}$ [35] M. I. Hussein, M. J. Leamy, M. Ruzzene, Dynamics of phononic materials and structures: Historical origins, recent progress, and future outlook, Appl. Mech. Rev. 66 (2014) 040802. 